\title{
Surface Activity of Alkoxy Ethoxyethyl $\beta$-D-Glucopyranosides
}

Yulin Fan, Fang Fu, Langqiu Chen, , Jiping Li, Jing Zhang

Key Laboratory of Environmentally Friendly Chemistry and Application of Ministry of Education, College of Chemistry, Xiangtan University, Xiangtan, Hunan, 411105, People's Republic of China

\section{Contents}

1. Synthesis routine of alkoxy ethoxyethyl $\beta$-D-glucopyranosides....................S2

2. General methods.............................................................S2

3. Synthesis of alkoxy ethoxyethyl $\beta$-D-glucopyranosides......................S2

4. Characterization of hexoxy ethoxyethyl 2,3,4,6- $O$-acetyl- $\beta$-D-glucopyranoside

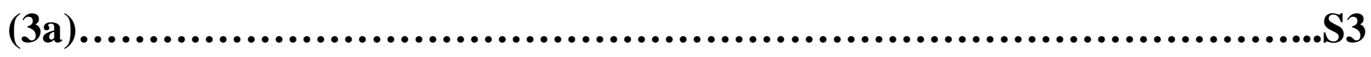

5. Characterization of alkoxy ethoxyethyl $\beta$-D-glucopyranosides (4a

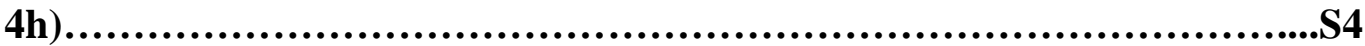




\section{Synthesis routine of alkoxy ethoxyethyl $\beta$-D-glucopyranosides}

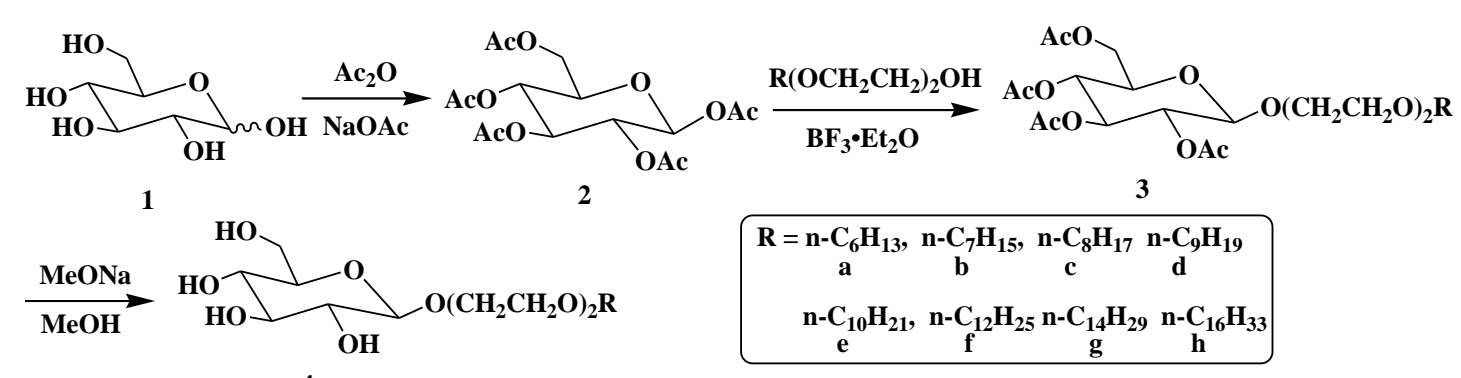

Figure S1. Synthesis of alkoxy ethoxyethyl $\beta$-D-glucopyranosides

\section{General methods}

The purity of all raw materials and reagents was analytical pure or chemical pure. All reaction processes were monitored by thin-layer chromatography (TLC). The TCL was performed on silica gel $\mathrm{HF}_{254}$ with detection by charring with a mixture of $30 \%(\mathrm{v} / \mathrm{v})$ sulfuric acid and methanol.

${ }^{1} \mathrm{H}$ NMR and HH COSY were determined with Bruker AVANCE III HD 400 $\mathrm{MHz}$ NMR Spectrometer with $\mathrm{D}_{2} \mathrm{O}$, DMSO- $d_{6}$, DMSO- $d_{6} / \mathrm{D}_{2} \mathrm{O}$, or chloroform- $d$ $\left(\mathrm{CDCl}_{3}\right)$ as the solvents and tetramethylsilane (TMS) as the internal standard. Optical rotations were obtained on a Perkin-Elmer model 341-MC automatic polarimeter for solutions in a 1-dm, jacketed cell. High resolution mass spectrum (HRMS) was determined with Thermo Scientific LTQ Orbitrap Xlwith ESI as ion resource.

\section{Synthesis of alkoxy ethoxyethyl $\beta$-D-glucopyranosides}

Diethylene glycol monohexyl ether $(15.66 \mathrm{~mL}, 76.92 \mathrm{mmol})$ was added to a stirred solution of 1,2,3,4,6-penta- $O$-acetyl- $\beta$-D-glucopyranose (2) (20.00 g, 51.23 mmol) from D-glucose (1) which was dissolved in methylene chloride $\left(\mathrm{CH}_{2} \mathrm{Cl}_{2}, 150\right.$ $\mathrm{mL})$ dried by $4 \AA$ molecular sieves. This mixture was stirred in an ice water bath for $10 \mathrm{~min}$, then the boron trifluoride etherate $\left(\mathrm{BF}_{3} \cdot \mathrm{Et}_{2} \mathrm{O}\right)(16.18 \mathrm{~mL}, 128.20 \mathrm{mmol})$ was added dropwise. The mixture was kept stirring for $5 \mathrm{~min}$, the ice water was removed and the reaction mixture continued to react for $9 \mathrm{~h}$ at the room temperature. The reaction progress was monitored by the TLC method (petroleum ether : ethyl acetate $=$ 
2:1) until the reaction completed. This reaction was quenched with saturated sodium carbonate aqueous solution and then extracted with $\mathrm{CH}_{2} \mathrm{Cl}_{2}$. The organic phase was dried over anhydrous sodium sulfate and then was filtered, the filtrate was concentrated under reduced pressure. The concentrate was purified by silica gel column chromatography (petroleum ether : ethyl acetate $=8: 1$ ) to obtain hexoxy ethoxyethyl 2,3,4,6-tetra- $O$-acetyl- $\beta$-D-glucopyranoside 3a as a colorless transparent syrup $(9.21 \mathrm{~g}$, yield, $34.5 \%)$.

Compound 3a (10.00 g, $19.21 \mathrm{mmol})$ was dissolved in methanol solution (65 $\mathrm{mL}$ ), the reaction solution was adjusted to $\mathrm{pH}=10$ with $10 \%$ sodium methoxide solution in methanol, the mixture was stirred at room temperature for $2 \mathrm{~h}$, the reaction progress was monitored by the TLC method (ethyl acetate : methanol =10:1) until the reaction completed and subsequently neutralized to $\mathrm{pH}=7.0$ with acetic acid. The mixture was concentrated under reduced pressure, the concentrate was purified by silica gel column chromatography (ethyl acetate : methanol $=50: 1$ ) to afford hexoxy ethoxyethyl $\beta$-D-glucopyranoside $4 \mathbf{a}$ (5.71 g, yield 84.4\%).

Other alkyloxy ethoxyethyl $\beta$-D-glucopyranosides $(\mathbf{4 b}, \mathbf{4 c}, \mathbf{4 d}, \mathbf{4 e}, \mathbf{4 f}, \mathbf{4 g}, \mathbf{4 h})$ were also synthesized in the same way.

\section{Characterization of hexoxy ethoxyethyl $2,3,4,6-O$-acetyl- $\beta$-D-glucopyranoside} (3a)

Yield, 34.5\%; $[\alpha]_{\mathrm{D}}^{20}-17.1^{\circ}\left(\mathrm{c} 1.0, \mathrm{CH}_{3} \mathrm{OH}\right) .{ }^{1} \mathrm{H}$ NMR $\left(\mathrm{CDCl}_{3}\right): \delta 5.21\left(\mathrm{dd}, 1 \mathrm{H}, J_{2,3}=\right.$ $\left.J_{3,4}=9.5 \mathrm{~Hz}, \mathrm{H}-3\right), 5.09\left(\mathrm{dd}, 1 \mathrm{H}, J_{4,5}=9.7 \mathrm{~Hz}, \mathrm{H}-4\right), 5.00(\mathrm{dd}, 1 \mathrm{H}, \mathrm{H}-2), 4.62(\mathrm{~d}, 1 \mathrm{H}$, $\left.J_{1,2}=8.0 \mathrm{~Hz}, \mathrm{H}-1\right), 4.26\left(\mathrm{dd}, 1 \mathrm{H}, J_{5,6}=4.7 \mathrm{~Hz}, J_{6,6}{ }^{\prime}=12.3 \mathrm{~Hz}, \mathrm{H}-6\right), 4.14\left(\mathrm{dd}, 1 \mathrm{H}, J_{5,6}\right.$ ' $=2.3 \mathrm{~Hz}, \mathrm{H}-6$ '), $3.92 \sim 3.97\left(\mathrm{~m}, 1 \mathrm{H}, \mathrm{CH}_{2} \mathrm{CH}_{2} \mathrm{O}\left(\mathrm{CH}_{2}\right)_{2} \mathrm{OC}_{6} \mathrm{H}_{13}\right), 3.72 \sim 3.79(\mathrm{~m}, 1 \mathrm{H}$, $\left.\mathrm{CH}_{2} \mathrm{CH}_{2} \mathrm{O}\left(\mathrm{CH}_{2}\right)_{2} \mathrm{OC}_{6} \mathrm{H}_{13}\right), 3.68 \sim 3.72(\mathrm{~m}, 1 \mathrm{H}, \mathrm{H}-5), 3.63 \sim 3.67(\mathrm{~m}, 2 \mathrm{H}$, $\left.\mathrm{CH}_{2} \mathrm{O}\left(\mathrm{CH}_{2}\right)_{2} \mathrm{OC}_{6} \mathrm{H}_{13}\right), 3.60 \sim 3.62\left(\mathrm{~m}, 2 \mathrm{H}, \mathrm{CH}_{2} \mathrm{CH}_{2} \mathrm{OC}_{6} \mathrm{H}_{13}\right), 3.54 \sim 3.57(\mathrm{~m}, 2 \mathrm{H}$, $\mathrm{CH}_{2} \mathrm{OC}_{6} \mathrm{H}_{13}$ ), 3.45 (t, $2 \mathrm{H}, J=6.8 \mathrm{~Hz}, \mathrm{OCH}_{2} \mathrm{C}_{5} \mathrm{H}_{11}$ ), 2.09 (s, 3H, Ac), 2.05 (s, 3H, Ac), 2.03 (s, 3H, Ac), 2.01 (s, 3H, Ac), $1.54 \sim 1.60\left(\mathrm{~m}, 2 \mathrm{H}, \mathrm{OCH}_{2} \mathrm{CH}_{2}\left(\mathrm{CH}_{2}\right)_{3} \mathrm{CH}_{3}\right), 1.26 \sim$ $1.35\left(\mathrm{~m}, 6 \mathrm{H}, \mathrm{O}\left(\mathrm{CH}_{2}\right)_{2}\left(\mathrm{CH}_{2}\right)_{3} \mathrm{CH}_{3}\right), 0.89\left(\mathrm{t}, 3 \mathrm{H}, \mathrm{J}=6.8 \mathrm{~Hz}, \mathrm{O}\left(\mathrm{CH}_{2}\right)_{5} \mathrm{CH}_{3}\right) . \mathrm{HRMS}(\mathrm{ESI})$ 
m/z: calculated for $\mathrm{C}_{24} \mathrm{H}_{40} \mathrm{O}_{12} \mathrm{Na}^{+}[\mathrm{M}+\mathrm{Na}]^{+}$, 543.24120; found 543.24182.

\section{Characterization of alkoxy ethoxyethyl $\beta$-D-glucopyranosides $(4 a \sim 4 h)$}

\subsection{Hexoxy ethoxyethyl $\beta$-D-glucopyranoside (4a)}

Yield, 84.4\%; $[\alpha]_{\mathrm{D}}^{20}-15.9^{\circ}\left(\mathrm{c} 1.0, \mathrm{CH}_{3} \mathrm{OH}\right) ;{ }^{1} \mathrm{H} \mathrm{NMR}\left(\mathrm{D}_{2} \mathrm{O}\right): \delta 4.47\left(\mathrm{~d}, 1 \mathrm{H}, J_{1,2}=\right.$ $7.9 \mathrm{~Hz}, \mathrm{H}-1), 4.02 \sim 4.07\left(\mathrm{~m}, 1 \mathrm{H}, \mathrm{CH}_{2} \mathrm{CH}_{2} \mathrm{O}\left(\mathrm{CH}_{2}\right)_{2} \mathrm{OC}_{6} \mathrm{H}_{13}\right), 3.90\left(\mathrm{dd}, 1 \mathrm{H}, J_{5,6}=2.1\right.$ $\left.\mathrm{Hz}, J_{6,6},=12.3 \mathrm{~Hz}, \mathrm{H}-6\right), 3.79 \sim 3.84\left(\mathrm{~m}, 1 \mathrm{H}, \mathrm{CH}_{2} \mathrm{CH}_{2} \mathrm{O}\left(\mathrm{CH}_{2}\right)_{2} \mathrm{OC}_{6} \mathrm{H}_{13}\right), 3.72 \sim 3.74$ $\left(\mathrm{m}, 2 \mathrm{H}, \mathrm{CH}_{2} \mathrm{O}\left(\mathrm{CH}_{2}\right)_{2} \mathrm{OC}_{6} \mathrm{H}_{13}\right), 3.63 \sim 3.70\left(\mathrm{~m}, 5 \mathrm{H}, \mathrm{H}-6\right.$ ', $\left.\mathrm{OCH}_{2} \mathrm{CH}_{2} \mathrm{OC}_{6} \mathrm{H}_{13}\right), 3.53$ (t, $\left.2 \mathrm{H}, J=6.8 \mathrm{~Hz}, \mathrm{OCH}_{2}\left(\mathrm{CH}_{2}\right)_{4} \mathrm{CH}_{3}\right), 3.47\left(\mathrm{dd}, 1 \mathrm{H}, J_{2,3}=J_{3,4}=9.1 \mathrm{~Hz}, \mathrm{H}-3\right), 3.41 \sim 3.46$ (m, 1H, H-5), 3.36 (dd, 1H, J4,5 = 9.3 Hz, H-4), 3.27 (dd, 1H, H-2), 1.53 1.60 (m, $\left.2 \mathrm{H}, \mathrm{OCH}_{2} \mathrm{CH}_{2}\left(\mathrm{CH}_{2}\right)_{3} \mathrm{CH}_{3}\right), 1.25 \sim 1.33\left(\mathrm{~m}, 6 \mathrm{H}, \mathrm{O}\left(\mathrm{CH}_{2}\right)_{2}\left(\mathrm{CH}_{2}\right)_{3} \mathrm{CH}_{3}\right), 0.85(\mathrm{t}, 3 \mathrm{H}, J=$ $\left.6.8 \mathrm{~Hz}, \mathrm{O}\left(\mathrm{CH}_{2}\right)_{5} \mathrm{CH}_{3}\right)$. HRMS(ESI) m/z: calculated for $\mathrm{C}_{16} \mathrm{H}_{32} \mathrm{O}_{8} \mathrm{Na}^{+}[\mathrm{M}+\mathrm{Na}]^{+}$, 375.19894; found 375.19928.

\subsection{Heptoxy ethoxyethyl $\beta$-D-glucopyranoside (4b)}

Yield, 87.7\%; $[\alpha]_{\mathrm{D}}^{20}-15.7^{\circ}\left(\mathrm{c} 1.0, \mathrm{CH}_{3} \mathrm{OH}\right) ;{ }^{1} \mathrm{H}$ NMR $\left(\mathrm{D}_{2} \mathrm{O}\right): \delta 4.50\left(\mathrm{~d}, 1 \mathrm{H}, J_{1,2}=\right.$ $7.9 \mathrm{~Hz}, \mathrm{H}-1), 4.05 \sim 4.10\left(\mathrm{~m}, 1 \mathrm{H}, \mathrm{CH}_{2} \mathrm{CH}_{2} \mathrm{O}\left(\mathrm{CH}_{2}\right)_{2} \mathrm{OC}_{7} \mathrm{H}_{15}\right), 3.93$ (dd, $1 \mathrm{H}, J_{5,6}=1.5$ $\left.\mathrm{Hz}, J_{6,6},=12.1 \mathrm{~Hz}, \mathrm{H}-6\right), 3.83 \sim 3.88\left(\mathrm{~m}, 1 \mathrm{H}, \mathrm{CH}_{2} \mathrm{CH}_{2} \mathrm{O}\left(\mathrm{CH}_{2}\right)_{2} \mathrm{OC}_{7} \mathrm{H}_{15}\right), 3.74 \sim 3.78$ (m, $\left.2 \mathrm{H}, \mathrm{CH}_{2} \mathrm{O}\left(\mathrm{CH}_{2}\right)_{2} \mathrm{OC}_{7} \mathrm{H}_{15}\right), 3.66 \sim 3.74$ (m, 5H, H-6', $\left.\mathrm{OCH}_{2} \mathrm{CH}_{2} \mathrm{OC}_{7} \mathrm{H}_{15}\right), 3.56$ (t, $\left.2 \mathrm{H}, J=6.8 \mathrm{~Hz}, \mathrm{OCH}_{2}\left(\mathrm{CH}_{2}\right)_{5} \mathrm{CH}_{3}\right), 3.51\left(\mathrm{dd}, 1 \mathrm{H}, J_{2,3}=J_{3,4}=9.1 \mathrm{~Hz}, \mathrm{H}-3\right), 3.45 \sim 3.48$ (m, 1H, H-5), 3.40 (dd, 1H, J4,5 = 9.3 Hz, H-4), 3.30 (dd, 1H, H-2), 1.56 1.63 (m, $\left.2 \mathrm{H}, \mathrm{OCH}_{2} \mathrm{CH}_{2}\left(\mathrm{CH}_{2}\right)_{4} \mathrm{CH}_{3}\right), 1.25 \sim 1.38\left(\mathrm{~m}, 8 \mathrm{H}, \mathrm{O}\left(\mathrm{CH}_{2}\right)_{2}\left(\mathrm{CH}_{2}\right)_{4} \mathrm{CH}_{3}\right), 0.88(\mathrm{t}, 3 \mathrm{H}, J=$ $\left.6.6 \mathrm{~Hz}, \mathrm{O}\left(\mathrm{CH}_{2}\right)_{6} \mathrm{CH}_{3}\right)$. HRMS(ESI) m/z: calculated for $\mathrm{C}_{17} \mathrm{H}_{34} \mathrm{O}_{8} \mathrm{Na}^{+}[\mathrm{M}+\mathrm{Na}]^{+}$, 389.21459; found 389.21466.

\subsection{Octoxyethoxy ethyl $\beta$-D-glucopyranoside (4c)}

Yield, 87.0\%; $[\alpha]_{\mathrm{D}}^{20}-16.0^{\circ}\left(\mathrm{c} 1.0, \mathrm{CH}_{3} \mathrm{OH}\right) ;{ }^{1} \mathrm{H}$ NMR $\left(\mathrm{D}_{2} \mathrm{O}\right): \delta 4.49\left(\mathrm{~d}, 1 \mathrm{H}, J_{1,2}=\right.$ $7.9 \mathrm{~Hz}, \mathrm{H}-1), 4.05 \sim 4.10\left(\mathrm{~m}, 1 \mathrm{H}, \mathrm{CH}_{2} \mathrm{CH}_{2} \mathrm{O}\left(\mathrm{CH}_{2}\right)_{2} \mathrm{OC}_{8} \mathrm{H}_{17}\right), 3.92\left(\mathrm{~d}, 1 \mathrm{H}, J_{6,6},=12.2\right.$ $\mathrm{Hz}, \mathrm{H}-6), 3.82 \sim 3.87\left(\mathrm{~m}, 1 \mathrm{H}, \mathrm{CH}_{2} \mathrm{CH}_{2} \mathrm{O}\left(\mathrm{CH}_{2}\right)_{2} \mathrm{O}\left(\mathrm{CH}_{2}\right)_{7} \mathrm{CH}_{3}\right), 3.75 \sim 3.79(\mathrm{~m}, 2 \mathrm{H}$, $\left.\mathrm{CH}_{2} \mathrm{O}\left(\mathrm{CH}_{2}\right)_{2} \mathrm{OC}_{8} \mathrm{H}_{17}\right), 3.65 \sim 3.75\left(\mathrm{~m}, 5 \mathrm{H}, \mathrm{H}-6\right.$ ', $\left.\mathrm{OCH}_{2} \mathrm{CH}_{2} \mathrm{OC}_{8} \mathrm{H}_{17}\right), 3.54$ (t, $2 \mathrm{H}, J=$ $\left.6.8 \mathrm{~Hz}, \mathrm{OCH}_{2}\left(\mathrm{CH}_{2}\right)_{6} \mathrm{CH}_{3}\right), 3.51\left(\mathrm{dd}, 1 \mathrm{H}, J_{2,3}=J_{3,4}=9.1 \mathrm{~Hz}, \mathrm{H}-3\right), 3.43 \sim 3.47(\mathrm{~m}, 1 \mathrm{H}$, 
H-5), $3.41\left(\mathrm{dd}, 1 \mathrm{H}, J_{4,5}=9.1 \mathrm{~Hz}, \mathrm{H}-4\right)$ ), $3.31(\mathrm{dd}, 1 \mathrm{H}, \mathrm{H}-2), 1.54 \sim 1.65(\mathrm{~m}, 2 \mathrm{H}$, $\left.\mathrm{OCH}_{2} \mathrm{CH}_{2}\left(\mathrm{CH}_{2}\right)_{5} \mathrm{CH}_{3}\right), 1.24 \sim 1.40\left(\mathrm{~m}, 10 \mathrm{H}, \mathrm{O}\left(\mathrm{CH}_{2}\right)_{2}\left(\mathrm{CH}_{2}\right)_{5} \mathrm{CH}_{3}\right), 0.89(\mathrm{t}, 3 \mathrm{H}, J=6.3$ $\left.\mathrm{Hz}, \mathrm{O}\left(\mathrm{CH}_{2}\right)_{7} \mathrm{CH}_{3}\right)$. HRMS(ESI) m/z: calculated for $\mathrm{C}_{18} \mathrm{H}_{36} \mathrm{O}_{8} \mathrm{Na}^{+}[\mathrm{M}+\mathrm{Na}]^{+}, 403.23024$; found 403.23068 .

\subsection{Nonoxy ethoxyethyl $\beta$-D-glucopyranoside (4d)}

Yield, 88.1\%; $[\alpha]_{\mathrm{D}}^{20}-15.9^{\circ}\left(\mathrm{c} 1.0, \mathrm{CH}_{3} \mathrm{OH}\right) ;{ }^{1} \mathrm{H}$ NMR $\left(\mathrm{D}_{2} \mathrm{O}\right): \delta 4.47\left(\mathrm{~d}, 1 \mathrm{H}, J_{1,2}=\right.$ $7.8 \mathrm{~Hz}, \mathrm{H}-1), 4.05 \sim 4.09\left(\mathrm{~m}, 1 \mathrm{H}, \mathrm{CH}_{2} \mathrm{CH}_{2} \mathrm{O}\left(\mathrm{CH}_{2}\right)_{2} \mathrm{OC}_{9} \mathrm{H}_{19}\right), 3.91\left(\mathrm{~d}, 1 \mathrm{H}, J_{6,6},=11.9\right.$ $\mathrm{Hz}, \mathrm{H}-6), 3.81 \sim 3.86\left(\mathrm{~m}, 1 \mathrm{H}, \mathrm{CH}_{2} \mathrm{CH}_{2} \mathrm{O}\left(\mathrm{CH}_{2}\right)_{2} \mathrm{OC}_{9} \mathrm{H}_{19}\right), 3.69 \sim 3.76(\mathrm{~m}, 5 \mathrm{H}$, $\mathrm{CH}_{2} \mathrm{OCH}_{2} \mathrm{CH}_{2} \mathrm{OC}_{9} \mathrm{H}_{19}, \mathrm{H}-6$ '), $3.62 \sim 3.66\left(\mathrm{~m}, 2 \mathrm{H}, \mathrm{CH}_{2} \mathrm{OC}_{9} \mathrm{H}_{19}\right), 3.51(\mathrm{t}, J=6.7 \mathrm{~Hz}$, $\left.\mathrm{OCH}_{2}\left(\mathrm{CH}_{2}\right)_{7} \mathrm{CH}_{3}\right), 3.43 \sim 3.51(\mathrm{~m}, 2 \mathrm{H}, \mathrm{H}-3, \mathrm{H}-5), 3.41\left(\mathrm{dd}, 1 \mathrm{H}, J_{3,4}=J_{4,5}=8.7 \mathrm{~Hz}\right.$, $\mathrm{H}-4), 3.31$ (dd, $\left.1 \mathrm{H}, J_{2,3}=8.6 \mathrm{~Hz}, \mathrm{H}-2\right), 1.55 \sim 1.65\left(\mathrm{~m}, 2 \mathrm{H}, \mathrm{OCH}_{2} \mathrm{CH}_{2}\left(\mathrm{CH}_{2}\right)_{6} \mathrm{CH}_{3}\right)$, $1.24 \sim 1.38\left(\mathrm{~m}, 12 \mathrm{H}, \mathrm{O}\left(\mathrm{CH}_{2}\right)_{2}\left(\mathrm{CH}_{2}\right)_{6} \mathrm{CH}_{3}\right), 0.89\left(\mathrm{t}, 3 \mathrm{H}, J=6.1 \mathrm{~Hz}, \mathrm{O}\left(\mathrm{CH}_{2}\right)_{8} \mathrm{CH}_{3}\right)$. HRMS(ESI) m/z: calculated for $\mathrm{C}_{19} \mathrm{H}_{38} \mathrm{O}_{8} \mathrm{Na}^{+}[\mathrm{M}+\mathrm{Na}]^{+}, 417.24589$; found 417.24612.

\subsection{Decoxy ethoxyethyl $\beta$-D-glucopyranoside (4e)}

Yield, 88.6\%; $[\alpha]_{\mathrm{D}}^{20}-14.4^{\circ}\left(\mathrm{c} 1.0, \mathrm{CH}_{3} \mathrm{OH}\right) ;{ }^{1} \mathrm{H}$ NMR $\left(\mathrm{D}_{2} \mathrm{O}\right): \delta 4.47\left(\mathrm{~d}, 1 \mathrm{H}, J_{1,2}=\right.$ $7.8 \mathrm{~Hz}, \mathrm{H}-1), 4.04 \sim 4.10\left(\mathrm{~m}, 1 \mathrm{H}, \mathrm{CH}_{2} \mathrm{CH}_{2} \mathrm{O}\left(\mathrm{CH}_{2}\right)_{2} \mathrm{OC}_{10} \mathrm{H}_{21}\right), 3.91\left(\mathrm{~d}, 1 \mathrm{H}, J_{6,6}=11.9\right.$ $\mathrm{Hz}, \mathrm{H}-6), 3.81 \sim 3.86\left(\mathrm{~m}, 1 \mathrm{H}, \mathrm{CH}_{2} \mathrm{CH}_{2} \mathrm{O}\left(\mathrm{CH}_{2}\right)_{2} \mathrm{OC}_{10} \mathrm{H}_{21}\right), 3.69 \sim 3.77(\mathrm{~m}, 5 \mathrm{H}$, $\mathrm{CH}_{2} \mathrm{OCH}_{2} \mathrm{CH}_{2} \mathrm{OC}_{10} \mathrm{H}_{21}, \mathrm{H}-6$ '), $3.62 \sim 3.66\left(\mathrm{~m}, 2 \mathrm{H}, \mathrm{CH}_{2} \mathrm{OC}_{10} \mathrm{H}_{21}\right), 3.48 \sim 3.53(\mathrm{~m}, 3 \mathrm{H}$, $\left.\mathrm{OCH}_{2}\left(\mathrm{CH}_{2}\right)_{8} \mathrm{CH}_{3}, \mathrm{H}-5\right), 3.42 \sim 3.45(\mathrm{~m}, 2 \mathrm{H}, \mathrm{H}-3, \mathrm{H}-4), 3.32\left(\mathrm{dd}, 1 \mathrm{H}, J_{2,3}=8.5 \mathrm{~Hz}\right.$, $\mathrm{H}-2), 1.56 \sim 1.63\left(\mathrm{~m}, 2 \mathrm{H}, \mathrm{OCH}_{2} \mathrm{CH}_{2}\left(\mathrm{CH}_{2}\right)_{7} \mathrm{CH}_{3}\right), 1.25 \sim 1.36(\mathrm{~m}, 14 \mathrm{H}$, $\left.\mathrm{O}\left(\mathrm{CH}_{2}\right)_{2}\left(\mathrm{CH}_{2}\right)_{7} \mathrm{CH}_{3}\right), 0.90\left(\mathrm{t}, 3 \mathrm{H}, J=6.1 \mathrm{~Hz}, \mathrm{O}\left(\mathrm{CH}_{2}\right)_{8} \mathrm{CH}_{3}\right) . \operatorname{HRMS}(\mathrm{ESI}) \mathrm{m} / \mathrm{z}$ : calculated for $\mathrm{C}_{20} \mathrm{H}_{40} \mathrm{O}_{8} \mathrm{Na}^{+}[\mathrm{M}+\mathrm{Na}]^{+}, 431.26154$; found 431.26181 .

\subsection{Dodecoxy ethoxyethyl $\beta$-D-glucopyranoside (4f)}

Yield, 87.5\%; $[\alpha]_{\mathrm{D}}^{20}-14.1^{\circ}\left(\mathrm{c} 1.0, \mathrm{CH}_{3} \mathrm{OH}\right) ;{ }^{1} \mathrm{H}$ NMR (DMSO-d6/D $\left.\mathrm{D}_{2} \mathrm{O}\right): \delta 4.15(\mathrm{~d}$, $\left.1 \mathrm{H}, J_{1,2}=7.8 \mathrm{~Hz}, \mathrm{H}-1\right), 3.80 \sim 3.86\left(\mathrm{~m}, 1 \mathrm{H}, \mathrm{CH}_{2} \mathrm{CH}_{2} \mathrm{O}\left(\mathrm{CH}_{2}\right)_{2} \mathrm{OC}_{12} \mathrm{H}_{25}\right), 3.63 \sim 3.66(\mathrm{~d}$, $\left.1 \mathrm{H}, J_{6,6},=11.7 \mathrm{~Hz}, \mathrm{H}-6\right), 3.50 \sim 3.59\left(\mathrm{~m}, 3 \mathrm{H}, \mathrm{CH}_{2} \mathrm{CH}_{2} \mathrm{OCH}_{2} \mathrm{CH}_{2} \mathrm{OC}_{12} \mathrm{H}_{25}\right), 3.46$ $3.50\left(\mathrm{~m}, 2 \mathrm{H}, \mathrm{CH}_{2} \mathrm{CH}_{2} \mathrm{OC}_{12} \mathrm{H}_{25}\right), 3.39 \sim 3.45$ (m, 3H, H-6', $\left.\mathrm{CH}_{2} \mathrm{OC}_{12} \mathrm{H}_{25}\right), 3.32$ (t, $J=$ $\left.6.6 \mathrm{~Hz}, 2 \mathrm{H}, \mathrm{OCH}_{2}\left(\mathrm{CH}_{2}\right)_{10} \mathrm{CH}_{3}\right), 3.15\left(\mathrm{dd}, 1 \mathrm{H}, J_{2,3}=J_{3,4}=9.0 \mathrm{~Hz}, \mathrm{H}-3\right), 3.08 \sim 3.13$ 
(m, 1H, H-5), 3.03 (dd, 1H, J4,5 = 9.1 Hz, H-4), 2.96 (dd, 1H, H-2), 1.37 1.46 (m, $\left.2 \mathrm{H}, \mathrm{OCH}_{2} \mathrm{CH}_{2}\left(\mathrm{CH}_{2}\right)_{9} \mathrm{CH}_{3}\right), 1.13 \sim 1.25\left(\mathrm{~m}, 18 \mathrm{H}, \mathrm{O}\left(\mathrm{CH}_{2}\right)_{2}\left(\mathrm{CH}_{2}\right)_{9} \mathrm{CH}_{3}\right), 0.80(\mathrm{t}, 3 \mathrm{H}, J=$ $\left.6.2 \mathrm{~Hz}, \mathrm{O}\left(\mathrm{CH}_{2}\right)_{11} \mathrm{CH}_{3}\right)$. HRMS(ESI) m/z: calculated for $\mathrm{C}_{22} \mathrm{H}_{44} \mathrm{O}_{8} \mathrm{Na}^{+}[\mathrm{M}+\mathrm{Na}]^{+}$, 459.29284; found 459.29327.

\subsection{Tetradecoxy ethoxyethyl $\beta$-D-glucopyranoside (4g)}

Yield, 89.4\%; $[\alpha]_{\mathrm{D}}^{20}-12.0^{\circ}$ (c 1.0, $\mathrm{CH}_{3} \mathrm{OH}$ ); ${ }^{1} \mathrm{H}$ NMR (DMSO-d6/D $2 \mathrm{O}$ ): $\delta 4.13(\mathrm{~d}$, $\left.1 \mathrm{H}, J_{1,2}=7.8 \mathrm{~Hz}, \mathrm{H}-1\right), 3.80 \sim 3.86\left(\mathrm{~m}, 1 \mathrm{H}, \mathrm{CH}_{2} \mathrm{CH}_{2} \mathrm{O}\left(\mathrm{CH}_{2}\right)_{2} \mathrm{OC}_{14} \mathrm{H}_{29}\right), 3.64(\mathrm{dd}, 1 \mathrm{H}$, $\left.J_{5,6}=1.7 \mathrm{~Hz}, J_{6,6^{\prime}}=11.8 \mathrm{~Hz}, \mathrm{H}-6\right), 3.51 \sim 3.58\left(\mathrm{~m}, 3 \mathrm{H}, \mathrm{CH}_{2} \mathrm{CH}_{2} \mathrm{OCH}_{2} \mathrm{CH}_{2} \mathrm{OC}_{14} \mathrm{H}_{29}\right)$, $3.48 \sim 3.50\left(\mathrm{~m}, 2 \mathrm{H}, \mathrm{CH}_{2} \mathrm{CH}_{2} \mathrm{OC}_{14} \mathrm{H}_{29}\right), 3.41 \sim 3.44\left(\mathrm{~m}, 2 \mathrm{H}, \mathrm{CH}_{2} \mathrm{OC}_{14} \mathrm{H}_{29}\right), 3.41$ (dd, $1 \mathrm{H}, J_{5,6}=5.9 \mathrm{~Hz}, \mathrm{H}-6$ '), $3.33\left(\mathrm{t}, J=6.6 \mathrm{~Hz}, 2 \mathrm{H}, \mathrm{OCH}_{2}\left(\mathrm{CH}_{2}\right)_{12} \mathrm{CH}_{3}\right), 3.13\left(\mathrm{dd}, 1 \mathrm{H}, J_{2,3}\right.$ $\left.=J_{3,4}=8.8 \mathrm{~Hz}, \mathrm{H}-3\right), 3.05 \sim 3.09(\mathrm{~m}, 1 \mathrm{H}, \mathrm{H}-5), 3.01\left(\mathrm{dd}, 1 \mathrm{H}, J_{4,5}=9.1 \mathrm{~Hz}, \mathrm{H}-4\right), 2.94$ (dd, 1H, H-2), $1.41 \sim 1.47\left(\mathrm{~m}, 2 \mathrm{H}, \mathrm{OCH}_{2} \mathrm{CH}_{2}\left(\mathrm{CH}_{2}\right)_{11} \mathrm{CH}_{3}\right), 1.17 \sim 1.26(\mathrm{~m}, 22 \mathrm{H}$, $\left.\mathrm{O}\left(\mathrm{CH}_{2}\right)_{2}\left(\mathrm{CH}_{2}\right)_{11} \mathrm{CH}_{3}\right), 0.82\left(\mathrm{t}, 3 \mathrm{H}, J=6.8 \mathrm{~Hz}, \mathrm{O}\left(\mathrm{CH}_{2}\right)_{13} \mathrm{CH}_{3}\right) . \mathrm{HRMS}(\mathrm{ESI}) \mathrm{m} / \mathrm{z}$ : calculated for $\mathrm{C}_{24} \mathrm{H}_{48} \mathrm{O}_{8} \mathrm{Na}^{+}[\mathrm{M}+\mathrm{Na}]^{+}, 487.32414$; found 487.32449.

\subsection{Hexadecoxy ethoxyethyl $\beta$-D-glucopyranoside (4h)}

Yield, 89.5\%; $[\alpha]_{\mathrm{D}}^{20}-11.5^{\circ}$ (c 1.0, $\left.\mathrm{CH}_{3} \mathrm{OH}\right) ;{ }^{1} \mathrm{H}$ NMR (DMSO-d6/D $\left.2 \mathrm{O}\right): \delta 4.13(\mathrm{~d}$, $\left.1 \mathrm{H}, J_{1,2}=7.8 \mathrm{~Hz}, \mathrm{H}-1\right), 3.79 \sim 3.84\left(\mathrm{~m}, 1 \mathrm{H}, \mathrm{CH}_{2} \mathrm{CH}_{2} \mathrm{O}\left(\mathrm{CH}_{2}\right)_{2} \mathrm{OC}_{16} \mathrm{H}_{33}\right), 3.64(\mathrm{dd}, 1 \mathrm{H}$, $\left.J_{5,6}=1.7 \mathrm{~Hz}, \quad J_{6,6}=12.0 \mathrm{~Hz}, \quad \mathrm{H}-6\right), \quad 3.50 \sim 3.57 \quad(\mathrm{~m}, \quad 3 \mathrm{H}$, $\left.\mathrm{CH}_{2} \mathrm{CH}_{2} \mathrm{OCH}_{2} \mathrm{CH}_{2} \mathrm{O}\left(\mathrm{CH}_{2}\right)_{15} \mathrm{CH}_{3}\right), 3.46 \sim 3.48\left(\mathrm{~m}, 2 \mathrm{H}, \mathrm{CH}_{2} \mathrm{CH}_{2} \mathrm{OC}_{16} \mathrm{H}_{33}\right), 3.40 \sim 3.44$ (m, 3H, $\mathrm{CH}_{2} \mathrm{OC}_{16} \mathrm{H}_{33}, \mathrm{H}-6$ '), 3.30 (t, $\left.2 \mathrm{H}, J=6.6 \mathrm{~Hz}, \mathrm{OCH}_{2}\left(\mathrm{CH}_{2}\right)_{14} \mathrm{CH}_{3}\right), 3.14(\mathrm{dd}, 1 \mathrm{H}$, $\left.J_{2,3}=J_{3,4}=8.7 \mathrm{~Hz}, \mathrm{H}-3\right), 3.05 \sim 3.11(\mathrm{~m}, 1 \mathrm{H}, \mathrm{H}-5), 3.04\left(\mathrm{dd}, 1 \mathrm{H}, J_{3,4}=J_{4,5}=9.5 \mathrm{~Hz}\right.$, $\mathrm{H}-4), 2.95\left(\mathrm{dd}, 1 \mathrm{H}, J_{1,2}=8.0 \mathrm{~Hz}, J_{2,3}=8.8 \mathrm{~Hz}, \mathrm{H}-2\right), 1.37 \sim 1.46(\mathrm{~m}, 2 \mathrm{H}$, $\left.\mathrm{OCH}_{2} \mathrm{CH}_{2}\left(\mathrm{CH}_{2}\right)_{13} \mathrm{CH}_{3}\right), 1.10 \sim 1.24\left(\mathrm{~m}, 26 \mathrm{H}, \mathrm{O}\left(\mathrm{CH}_{2}\right)_{2}\left(\mathrm{CH}_{2}\right)_{13} \mathrm{CH}_{3}\right), 0.78(\mathrm{t}, 3 \mathrm{H}, J=$ $\left.6.7 \mathrm{~Hz}, \mathrm{O}\left(\mathrm{CH}_{2}\right)_{15} \mathrm{CH}_{3}\right)$. HRMS(ESI) $\mathrm{m} / \mathrm{z}$ : calculated for $\mathrm{C}_{26} \mathrm{H}_{52} \mathrm{O}_{8} \mathrm{Na}^{+}[\mathrm{M}+\mathrm{Na}]^{+}$, 515.35544; found 515.35565. 